\title{
Coherent Meson Production in the NOMAD Experiment
}

\section{Chris Kullenberg*}

Joint Institute for Nuclear Research

E-mail: chriskullenberg@gmail.com

\section{Sanjib R. Mishra}

University of South Carolina

E-mail: sanjib0704@gmail.com

\begin{abstract}
We presented the measurements of coherent $\pi^{0}, \rho^{+}$and $\rho^{0}$ meson production in the NOMAD experiment. The excellent angular and momentum resolutions of the NOMAD detector - based upon a low density design with $\rho \sim 0.1 \mathrm{~g} / \mathrm{cm}^{3}-$ combined with a nuclear target predominantly made of carbon, result in an efficient selection of coherent processes with small background levels. Coherent processes in neutrino interactions are characterized by the production of forward mesons with respect to the beam direction with very small momentum transfer to the nucleus. These interactions can serve as a test of the structure of the weak current, including PCAC and CVC, which are used to model the coherent processes.
\end{abstract}

European Physical Society Conference on High Energy Physics - EPS-HEP2019 -

10-17 July, 2019

Ghent, Belgium

*Speaker. 


\section{Introduction}

In coherent neutrino production of mesons all nucleons within the target participate in the interaction. In order for this to occur there must be a small momentum transfer to the nucleus, else a single nucleon may be ejected, the nucleus must recoil intact in its ground state, and there must be no transfer of quantum numbers, else a nucleon is singled out and coherence is broken. The mesons produced will tend to have a small angle relative to the incoming neutrino direction. Feynman diagrams for coherent production of pions and rho mesons can be seen in figure 1. These interactions are interesting because they can provide a better understanding of the composition of the weak current, as they both proceed via different mechanisms.

Coherent pion production is an axial-vector dominated process. It can be shown that for small $Q^{2}$ (negative of the square of the 4-momentum transfer to the hadronic system), Adler's relation allows us to relate the cross-section of coherent neutrino production of pions to the strong coherent scattering of pions on nuclei with a simple multiplicative factor [1]. NOMAD has tested this modeling of the process at high $v$ (energy transferred to the hadronic system) with a measurement of coherent $\pi^{0}$ production [2]. The results were found to be consistent with Rein and Sehgal's prediction, though there are various parameters within the model that require more data to determine. We hope to eventually improve on this measurement by using additional signal channels and updated methods.

Coherent rho production, however, proceeds through the action of the weak vector current. Piketty and Stodolky first proposed the use of vector meson dominance within the lepto sector, analogous to its successful application to the photo-production of vector mesons [3]. Using vector meson dominance (VMD) and conservation of vector current (CVC) coherent rho production can also be expressed in terms of meson-nucleus scattering:

$$
\frac{d^{3} \sigma\left(v_{\mu} \mathcal{A} \rightarrow \mu^{-} \rho^{+} \mathcal{A}\right)}{d Q^{2} d v d t}=\frac{G_{F}^{2} f_{\rho}^{2}}{4 \pi^{2}} \frac{|q|}{E_{v}^{2}}\left(\frac{Q}{Q^{2}+m_{\rho}^{2}}\right)^{2} \frac{(1+\varepsilon R)}{1-\varepsilon}\left[\frac{d \sigma^{T}\left(\rho^{+} \mathcal{A} \rightarrow \rho^{+} \mathcal{A}\right)}{d t}\right]
$$

where $G_{F}$ is the weak coupling constant, $f_{\rho}$ is the $\rho$ form factor and is related to the corresponding charged current scattering factor, $f_{\rho}^{ \pm}=f_{\rho^{0}}^{\gamma} \sqrt{2} \cos \theta_{C}$, where $\theta_{C}$ is the Cabibbo angle and $f_{\rho}^{\gamma}=m_{\rho}^{2} / \gamma_{\rho}$ is the coupling of $\rho^{0}$ to photon $\left(\gamma_{\rho}^{2} / 4 \pi=2.4 \pm 0.1\right)$. The polarization parameter is defined as $\varepsilon=\frac{4 E_{v} E_{\mu}-Q^{2}}{4 E_{v} E_{\mu}+Q^{2}+2 v^{2}}$, and $R=\frac{d \sigma^{L} / d t}{d \sigma^{T} / d t}$ with $\sigma^{L}$ and $\sigma^{T}$ as the longitudinal and transverse $\rho$-nucleus cross-sections, respectively.

NOMAD studied coherent production of $\rho^{+}$, and found the results to be consistent with the VMD model with $\mathrm{R}=0$ (small contribution from longitudinal component) [4]. Coherent $\rho^{+}$production is directly related to $\rho^{0}$ production through a simple factor based on the weak mixing angle, $\theta_{W}$, with a value of $\sim 0.15$ :

$$
\frac{d^{3} \sigma\left(v_{\mu} \mathcal{A} \rightarrow v_{\mu} \rho^{0} \mathcal{A}\right)}{d Q^{2} d v d t}=\frac{1}{2}\left(1-2 \sin ^{2} \theta_{W}\right)^{2}\left[\frac{d^{3} \sigma\left(v_{\mu} \mathcal{A} \rightarrow \mu^{-} \rho^{+} \mathcal{A}\right)}{d Q^{2} d v d t}\right]
$$

A measurement of coherent $\rho^{0}$ production will further serve to test our modeling of vector meson production in weak interactions (namely CVC and the use of VMD in lepto-production), 
and function as a cross-check of the charged current channel. Here we present the preliminary results of our coherent $\rho^{0}$ production analysis.
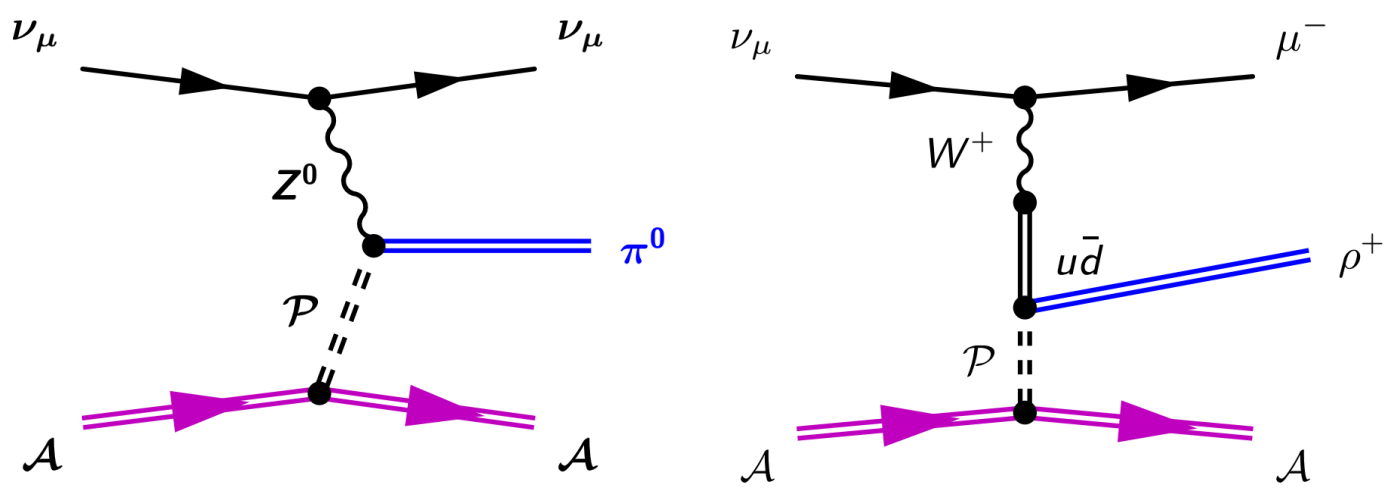

Figure 1: Feynman diagrams for coherent neutrino production of $\pi^{0}$ (left) and $\rho^{+}$(right), with $\mathcal{A}$ representing the nuclear target and $\mathcal{P}$ the particle performing the momentum transfer to the target (assumed to be a Pomeron).

\section{The NOMAD Detector}

NOMAD was primarily designed to search for $v_{\mu} \rightarrow v_{\tau}$ oscillations. But its excellent position and momentum resolution $(\delta r<200 \mu m, \delta p \sim 3.5 \% @ p<10 \mathrm{GeV} / \mathrm{c})$ make it an prime candidate for cross-section measurements. At the heart of NOMAD are its drift chambers (DC), providing fine-grained tracking while acting as the primary target (see figure 2). The DC was contained within a $0.4 \mathrm{~T}$ magnetic field, allowing for the unambiguous separation of charges. It also contained transition radiation detectors and muon chambers for particle ID, as well as front and rear calorimeters. NOMAD was exposed to the CERN SPS neutrino beam, composed primarily of muon neutrinos with an average energy of $24 \mathrm{GeV}$. For details on NOMAD's construction and performance see [5].

Over its lifetime NOMAD collected 1.44 million $v_{\mu}$ charged current events, and will continue to be one of the highest quality collections of neutrino data until the commencement of the next generation of tracking neutrino detectors (DUNE).

\section{Coherent $\rho^{0}$ Event Selection}

The signature for coherent $\rho^{0}$ production is a pair of charged pions from the $\rho$ decay and nothing else in the detector. A candidate signal event from data can be seen in figure 3. Backgrounds will include two-track events from neutral current deep inelastic scattering (NC-DIS), two-track charged current DIS (CC-DIS) events, where the muon has not been identified, and outside background events $(\mathrm{OBG})$, composed largely of neutral kaons originating from upstream and decaying in the drift chambers.

The distinguishing kinematic feature of coherent neutrino production is the relative co-linearity of the emerging meson with the neutrino beam. For neutral current interactions this forwardness is characterized by $\zeta=E(1-\cos \theta)$, where $E$ is the energy of the emerging meson, and $\theta$ its angle w.r.t. the neutrino beam. 


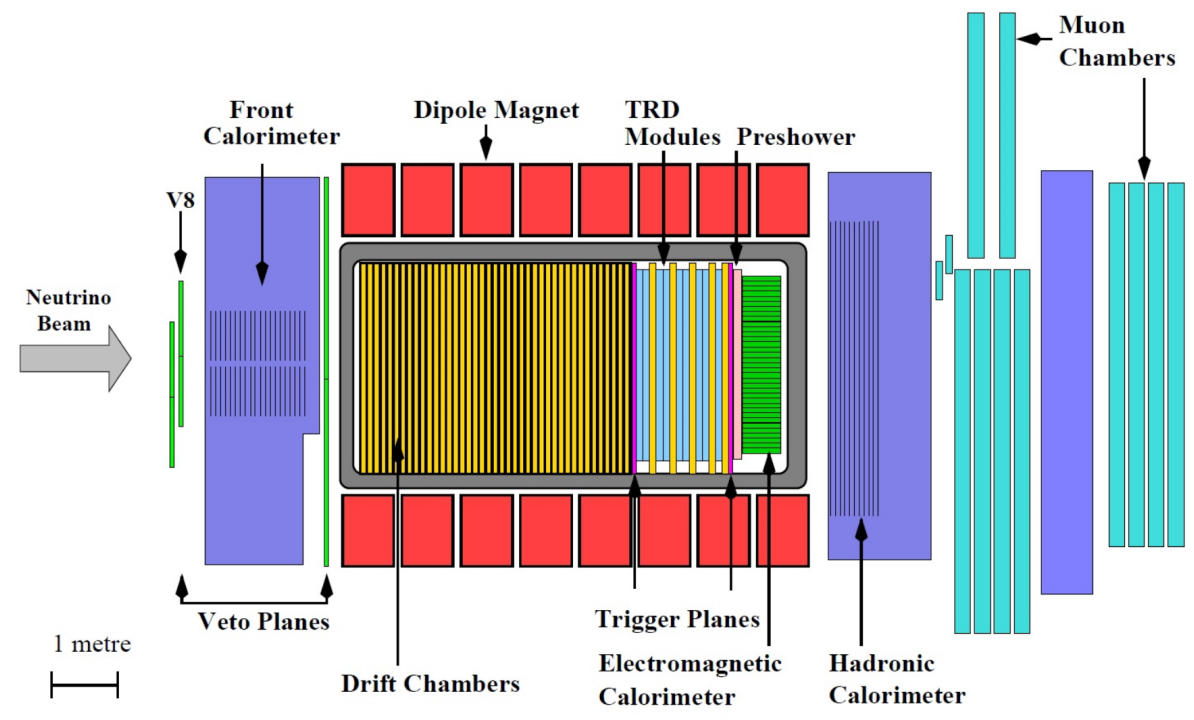

Figure 2: A side view of the NOMAD detector.

We simulate coherent $\rho^{0}$ production using the relationship in equation 1.1, and we use Rein and Sehgal's method to model the meson-nucleus scattering cross-section [1]. For the DIS background contributions we use NEGLIB (NOMAD Event Generator Library based on LEPTO 6.1 and JETSET 7.4 with GEANT-3 for detector response) to simulate neutrino interactions in our detector. For OBG we use data themselves to establish the shape of the background. We first identify data events with a reconstructed primary vertex upstream of the drift chambers and with a two-track $V^{0}$ (vertex with no net charge) reconstructed within the detector. We then ignore the primary vertex and use the kinematics of the track pair to simulate the shape of the OBG contribution.

Additionally we realized early in the analysis that the shape of the NC-DIS MC needed calibration, particularly the $\zeta$ distribution, which is the most important variable in the analysis. To correct the shape we again use an independent data sample by selecting the charged current version of our topology, having and outgoing muon and two oppositely charged tracks. We then ignore the muon track (see figure 3) and use the properties of the remaining two-track system to form kinematic correction functions by simply taking the ratio of Data/MC in the 3-track sample. We then apply the CC-DIS sample's correction functions to the NC-DIS MC background sample used in the analysis. If we did not correct our default MC it would result in an overestimation of our signal contribution.

\section{Results}

After using data to constrain our background shapes we are free to measure the signal contribution. We normalize the DIS within a kinematic region with little signal contribution $\left(\zeta_{\pi \pi}>0.075\right)$ using the angle between the two tracks in a plane perpendicular to the neutrino beam direction $\left(\phi_{12}\right)$. We then normalize the OBG background using the number of events within the kaon mass peak $\left(0.47 \leq M_{\pi \pi} \leq 0.53 \mathrm{GeV} / \mathrm{c}^{2}\right)$. After setting the level of background contribution we measure the signal using $\zeta_{\pi \pi}$ within the $\rho$ mass range $\left(0.6 \leq M_{\pi \pi} \leq 1.0 \mathrm{GeV} / c^{2}\right)$. See figure 5 for 


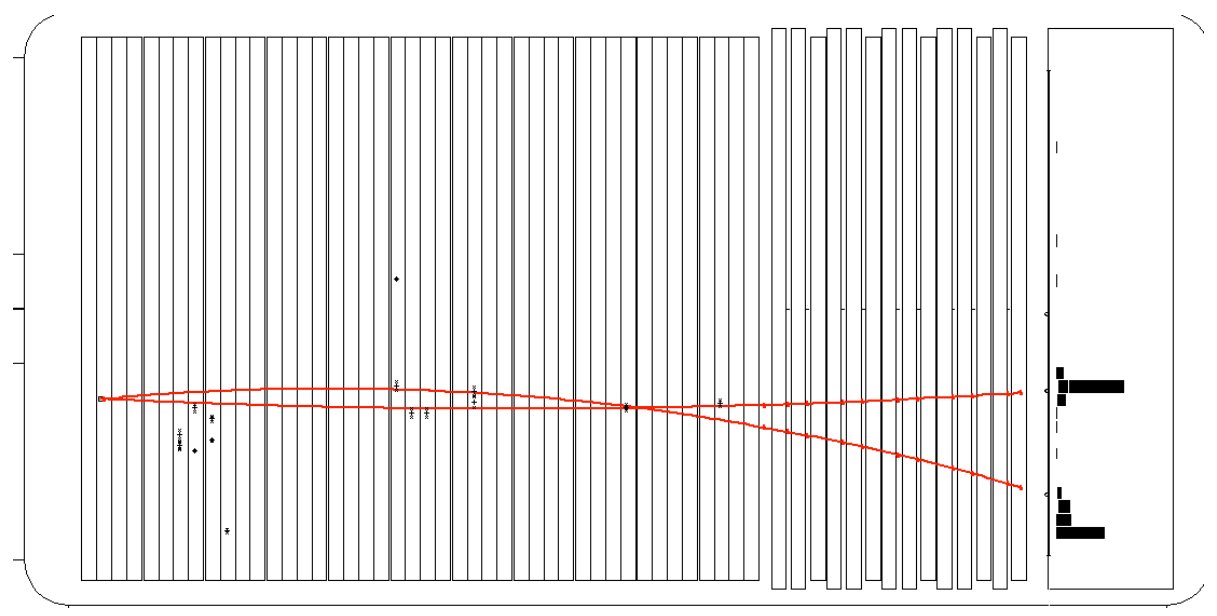

Figure 3: A Coherent $\rho^{0}$ candidate event from NOMAD data, having $\zeta_{\pi \pi}=0.024$ and $M_{\pi \pi}=0.61 \mathrm{GeV} / \mathrm{c}^{2}$.

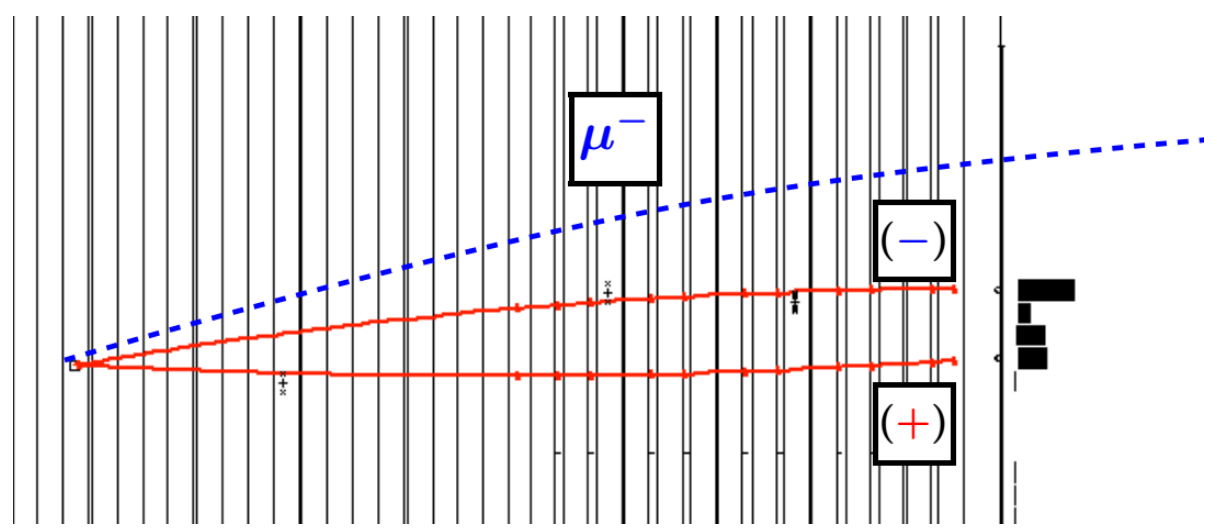

Figure 4: A diagram representing the DS method. We choose 3-track events having a muon (CC-DIS), ignore the muon track, and use the remaining two-track system to correct the MC kinematics in our background sample (NC-DIS).

examples of the kinematic variables used to measure the contributions from each component. The normalization process is then iterated until convergence.

We estimate the systematic error contribution from the DS method to be nearly $11 \%$, which is the largest contributor. This is unavoidable, as an accurate background $\zeta$ shape is crucial to our measurement. The other main contribution comes from the largest background, NC-DIS, with small additions from the remaining backgrounds, giving us a total systematic error of $13.6 \%$.

There is a clear excess over the data within the rho mass range, as can bee seen in figure 6 . We report a preliminary observation of $560 \pm 105$ (Stat.) $\pm 76\left(\right.$ Syst.) fully corrected Coherent- $\rho^{0}$ events with a total error of $23 \%$. The rate with respect to $\left(1.44 * 10^{6}\right) v_{\mu}$-CC events is $(3.89 \pm$ $0.9) \times 10^{-4}$. 

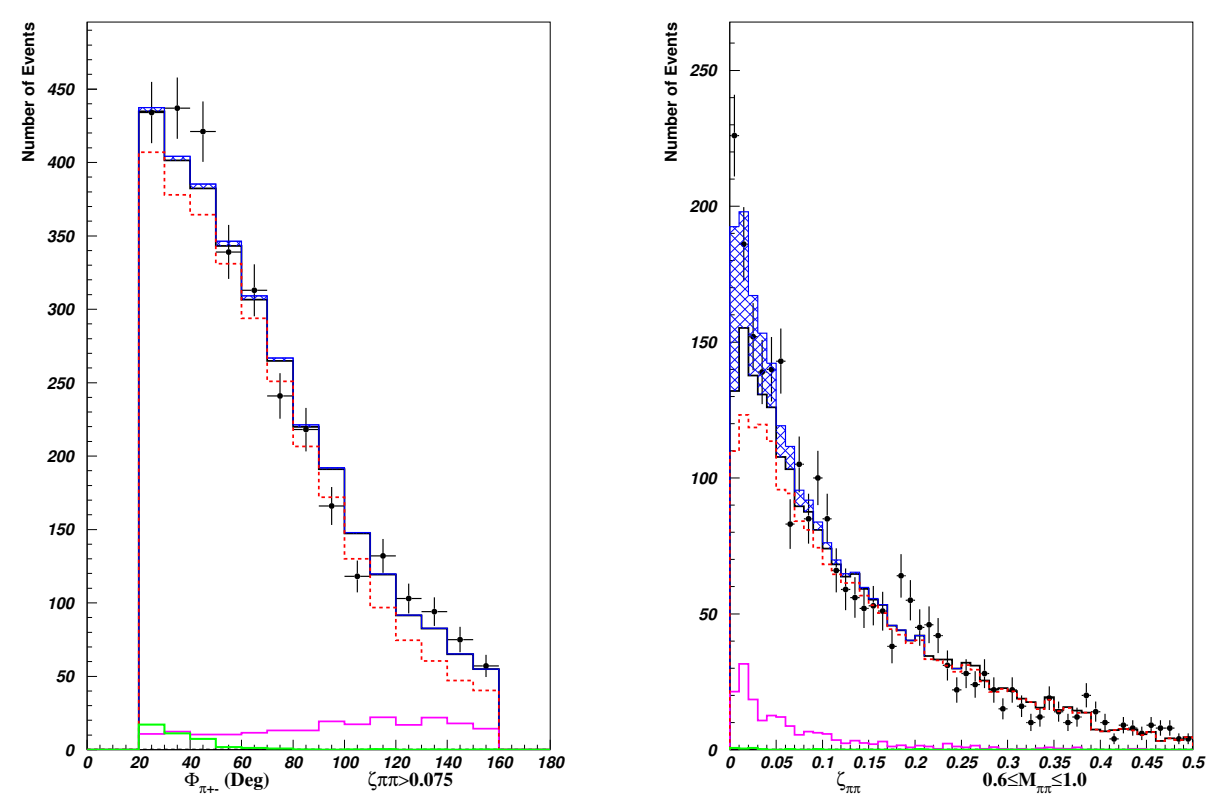

Figure 5: Here we show the variables used to measure the contributions from the main background (NC-DIS in red dashes) and the signal ( $\mathrm{Coh} \rho^{0}$ in blue, filled hatch). Other background contributions include CC-DIS (solid magenta) and OBG (solid green). NC-DIS is normalized on $\phi_{12}$ (angle between the tracks in the plane perpendicular to the neutrino beam) with large $\zeta_{\pi \pi}$ (outside the signal region). The signal is normalized on $\zeta_{\pi \pi}$ within the rho mass range.

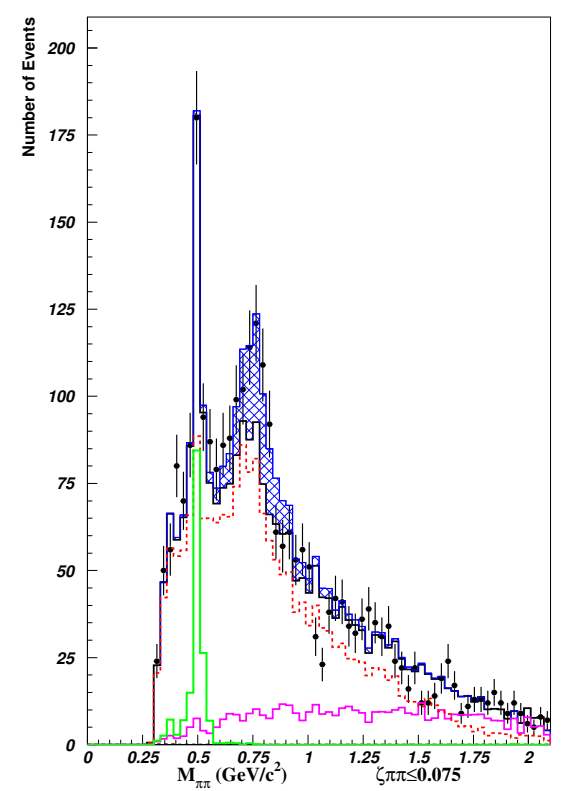

Figure 6: Here we show the final mass plot within the signal range $\left(\zeta_{\pi \pi}<0.075\right)$. A clear excess over background can be seen within the rho mass peak. $\operatorname{Coh} \rho^{0}$ is in blue hatch, NC-DIS in dashed red, CC-DIS in solid magenta, and OBG in solid green. 


\section{References}

[1] D. Rein and L. M. Sehgal, Coherent piO Production in Neutrino Reactions, Nucl. Phys. B223 (1983) 29.

[2] NOMAD collaboration, A Measurement of Coherent Neutral Pion Production in Neutrino Neutral Current Interactions in NOMAD, Phys. Lett. B682 (2009) 177 [0 910.0062 ].

[3] C. A. Piketty and L. Stodolsky, Diffraction model of high-energy leptonic interactions, Nucl. Phys. B15 (1970) 571.

[4] NOMAD collaboration, Neutrino induced charged-current coherent $\rho$ production, in 15th International Workshop on Neutrino Factories, Super Beams and Beta Beams (NuFact2013) Beijing, China, August 19-24, 2013, 2013, 1310 .8547.

[5] NOMAD collaboration, The NOMAD experiment at the CERN SPS, Nucl. Instrum. Meth. A404 (1998) 96. 